\title{
Statistical Constraints on Synaptic Plasticity
}

The synaptic basis for learning suggested by Hebb (1949), Marr's theory (1969) for motor learning in the cerebellum, and Stent's mechanism (1973) for synaptic depression in visual cortex depend on coincidences between presynaptic and postsynaptic events. Two statistical constraints on this class of mechanisms will be examined here: the first concerns the problem of synaptic saturation from chance coincidences, and the second is the problem of random fluctuations. An alternative hypothesis for synaptic plasticity is suggested which minimizes the first problem.

\section{CEREBELLUM}

The cerebellum is an especially promising model system for studying synaptic plasticity in vertebrates, first because of its simple, repetitive, well-studied structure (Eccles, Ito \& Szentágothai, 1967), and secondly because of recent evidence for its involvement in motor learning: the vestibulo-ocular reflex can be modified by visual experience, but this plasticity is entirely abolished by lesions to the cerebellar flocculus (Ito, 1975; Robinson, 1976).

Purkinje cells, which provide the only output from the cerebellum, receive information about the internal state of the nervous system and the external sensory environment through parallel fibers. Each Purkinje cell also receives multiple synaptic contacts from the single climbing fiber which entwines its dendritic tree. These two main inputs to Purkinje cells are illustrated in Fig. 1.

Marr (1969) has proposed a theory for motor learning in the cerebellum which predicts that "the synapses from parallel fibers to Purkinje cells are facilitated by the conjunction of presynaptic and climbing fiber (or postsynaptic) activity". Marr's prediction is related to Hebb's suggestion (1949) that the strength of a plastic synapse may be increased by simultaneous presynaptic and postsynaptic discharges. Some of these coincident events take place purely by chance; since accidental coincidences are unrelated to an animal's experience, they can have little or no adaptive value. Moreover, unless some means exist for weakening the plastic synapse, continual random coincidences inexorably push it to maximum strength. A plastic synapse whose strength can be flexibly adjusted within its range should therefore be capable of long-term depression as well as long-term 


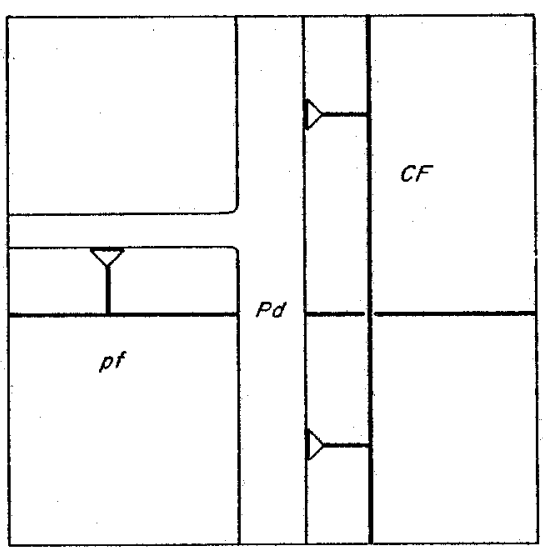

FIG. 1. Schematic illustration of a cerebellar Purkinje cell dendrite $P d$ (with a dendritic branchlet), a climbing fiber $C F$ (entwining the dendritic trunk), and a parallel fiber $p f$ (passing through the dendritic tree perpendicular to the plane of arborization), based on Eccles, Ito \& Szentagothai (1967). The synapse between the parallel fiber and Purkinje cell is presumed to vary in strengh. The hypothesis considered is that the synaptic strength cell is presumed to vary in strength. The hypothesis considered is that the synaptic strength in the parallel fiber and the climbing fiber (Sejnowski, 1977).

facilitation, and the condition for weakening the synapse should be as specific as that for strengthening it-otherwise the information stored as the synaptic strength is lost.

Various mechanisms for overcoming synaptic saturation could be put forward. For example, von der Malsburg (1973) has suggested that, following synaptic strengthening, the strength of all plastic synapses on a neuron should be normalized so that the total synaptic input remains constant. Uttley (1976) has given a learning algorithm based on conditional probabilities which results in zero total synaptic input to a neuron. The following hypothesis, which arose naturally in a recent probabilistic analysis of interacting neurons (Sejnowski, 1976, 1977), minimizes the problem of synaptic saturation without placing any restrictions on the total synaptic input, and thereby allowing maximum flexibility.

\section{Balanced synaptic plasticity}

Let us assume that the synapse between a parallel fiber and Purkinje cell dendrite (shown in Fig. 1) is plastic, and that the change in its synaptic strength is proportional to the covariance between discharges of the parallel fiber and the climbing fiber: then the synapse increases in strength when the discharges are positively correlated, decreases in strength when the discharges are negatively correlated, and maintains a constant average strength when the discharges are uncorrelated.

This hypothesis puts constraints on possible mechanisms for synaptic plasticity. Suppose that the synaptic strength increases by a small amount $\alpha$ whenever a coincidence occurs between discharges in the climbing fiber and plastic synapse within time interval $\tau / 2$. Neglecting multiple coincidences, the accidental or chance coincidence rate for independently occurring discharges is $R_{c} R_{p} \tau$, with $R_{c}$ and $R_{p}$ the average firing rates of the climbing fiber and plastic synapse, respectively.

Chance strengthening must be balanced by a mechanism which weakens the synapse. For example, Stent (1973) has postulated that neurotransmitter receptors are removed from a plastic synapse when the postsynaptic membrane repeatedly discharges in the absence of a presynaptic discharge. Stent further suggested that the mechanism might account for developmental plasticity in the cat visual cortex.

Assume that the plastic synapse diminishes in strength by $\beta$ whenever the climbing fiber discharges without a discharge in the plastic synapse within $\tau_{p} / 2$. If multiple coincidences within this time interval are negligible, then the condition for balance between strengthening and weakening when the inputs are uncorrelated is:

$$
\beta\left(1-R_{p} \tau_{p}\right)=\alpha R_{p} \tau .
$$

This condition for balance may fail to be satisfied when the strength of the plastic synapse is at either its minimum or maximum.

Thus, the plastic synapse is modified whenever the climbing fiber discharges; whether the synapse is strengthened or weakened depends on whether or not it discharges in coincidence with the climbing fiber. Consider the case when $\tau_{p}$ is sufficiently small so that $R_{p} \tau_{p} \ll 1$. Then the ratio of weakening to strengthening is, by equation (1), proportional to the firing rate of the plastic synapse: $\beta / \alpha=R_{p} \tau$. A substance present in the synaptic cleft in proportion to the average firing rate of the synapse (such as a degradation product of the neurotransmitter) could serve as agent in satisfying this condition for balance.

An alternative way to balance the strengthening is to have the synaptic strength diminish by $\beta$ whenever the plastic synapse discharges without a discharge of the climbing fiber within $\tau_{c} / 2$. The corresponding balance condition for uncorrelated inputs is:

$$
\beta\left(1-R_{c} \tau_{c}\right)=\alpha R_{c} \tau
$$




\section{Random fluctuations}

The number of coincidences and anti-coincidences is subject to random fluctuations. For stationary independent inputs over a time $T$, the synaptic strength, within its range, will undergo a random walk with standard deviation

$$
\sigma(T)=\left[\left(\alpha^{2}+\alpha \beta\right) R_{c} R_{p} \tau T\right]^{1 / 2}
$$

around its mean strength. Random fluctuations can be minimized by regulating the time during which the plastic synapse is sensitive to modification -a slow and diffuse chemical system would suffice.

Let us apply these general statistical constraints specifically to the cerebellum. Under typical conditions the firing rate of a parallel fiber is $R_{\mathrm{p}} \sim 50$ per $\mathrm{s}$ and the firing rate of a climbing fiber is $R_{c} \sim 1$ per $\mathrm{s}$. If we assume that the coincidence "window" for strengthening is $\tau \sim 2 \mathrm{~ms}$ (comparable to the time course of an action potential), and if $\tau_{p} \leqslant 20 \mathrm{~ms}$ (so that $R_{p} \tau_{p} \ll 1$ ) then the balance condition (1) becomes $\beta / \alpha \sim 0 \cdot 1$; that is, the size of a decrement from a single anti-coincidence must be approximately one-tenth the size of an increment from a single coincidence. Under the same steady-state conditions the standard deviation in equation (3) owing to random fluctuations over one hour is $\sigma \sim 20 \alpha$. In comparison, the maximum non-random plastic change of the synaptic strength in one hour is $3600 \alpha$ (which occurs when every climbing-fiber discharge is in coincidence with a discharge of the plastic synapse); thus, the contribution from random fluctuations is a relatively small fraction of the range within which the strength of a synapse could be adjusted in one hour. Random fluctuations are nevertheless cumulative and must be considered a serious long-term noise problem.

\section{ConClusion}

If the above balance condition is satisfied, then the average strength of the plastic synapse cannot be altered by simply increasing the firing rate of the climbing fiber or the parallel fiber: the strength of the synapse can be systematically altered only through correlated discharges, and the synapse is then either strengthened or weakened depending on the sign of the covariance. This hypothesis has several consequences: first, the problem of synaptic saturation from chance coincidences is overcome; second, the entire dynamic range of synaptic strength is always accessible; and third, complex modulatory motor patterns are flexibly and efficiently stored in the cerebellum (Sejnowski, 1977). A direct test of the predicted synaptic plasticity in vertebrates is at present a difficult experiment; however, the general statistical constraints considered here apply equally well to invertebrates, and similar plasticity may occur in a more favorable preparation.

I am especially grateful to Alan Gelperin for helpful discussions.

Joseph Henry Laboratories,

Princeton University,

Princeton, New Jersey 08540, U.S.A.

(Received 20 December 1976, and in revised form 27 April 1977)

\section{REFERENCES}

EcCles, J. C., Ito, M. \& Szentágothai, J. (1967). The Cerebellum as a Neuronal Machine. Heidelberg: Springer.

Hens, D. O. (1949). The Organization of Behavior. New York: Wiley.

Ito, M. (1975). In The Nervous System (D. H. Tower, ed.), Vol. 1, pp. 245-251. New York: Raven Press.

MALSBURG, C. VON DER (1973). Kybernetik 14, 85.

MARR, D. (1969). J. Physiol., Lond. 202, 437.

RoBinson, D. A. (1976). J. Neurophysiol. 39, 954.

SEjNowski, T. J. (1976). Biol. Cybernet. 22, 203.

SeJNowsKi, T. J. (1977). J. math. Biol. (In press.)

STENT, G. S. (1973). Proc. natn. Acad. Sci., U.S.A. 70, 997.

UTTLEY, A. M. (1976). Brain Res. 102, 23. 